\title{
BALANCE AND PATTERN DISTRIBUTION OF SEQUENCES DERIVED FROM PSEUDORANDOM SUBSETS OF $\mathbb{Z}_{q}$
}

\author{
HuAning Liu ${ }^{1}$ - ARne WinterhoF ${ }^{2}$ \\ ${ }^{1}$ Research Center for Number Theory and Its Applications \\ ${ }^{2}$ Johann Radon Institute for Computational and Applied Mathematics
}

Dedicated to the memory of Reinhard Winkler

ABSTRACT. Let $q$ be a positive integer and

with

$$
\mathcal{S}=\left\{x_{0}, x_{1}, \ldots, x_{T-1}\right\} \subseteq \mathbb{Z}_{q}=\{0,1, \ldots, q-1\}
$$

$$
0 \leq x_{0}<x_{1}<\cdots<x_{T-1} \leq q-1 .
$$

We derive from $\mathcal{S}$ three (finite) sequences:

(1) For an integer $M \geq 2$ let $\left(s_{n}\right)$ be the $M$-ary sequence defined by

$$
s_{n} \equiv x_{n+1}-x_{n} \bmod M, \quad n=0,1, \ldots, T-2 .
$$

(2) For an integer $m \geq 2$ let $\left(t_{n}\right)$ be the binary sequence defined by

$$
t_{n}= \begin{cases}1 & \text { if } 1 \leq x_{n+1}-x_{n} \leq m-1, \quad n=0,1, \ldots, T-2 . \\ 0, & \text { otherwise, }\end{cases}
$$

(3) Let $\left(u_{n}\right)$ be the characteristic sequence of $\mathcal{S}$,

$$
u_{n}=\left\{\begin{array}{ll}
1 & \text { if } n \in \mathcal{S}, \\
0, & \text { otherwise },
\end{array} \quad n=0,1, \ldots, q-1 .\right.
$$

(C) 2021 BOKU-University of Natural Resources and Life Sciences and Mathematical Institute, Slovak Academy of Sciences.

2010 Mathematics Subject Classification: 11K45, 94A55, 11T71, $11 \mathrm{Z} 05$.

Keywords: sequence, pseudorandom subset, balance, pattern distribution, correlation measure.

The first author is supported by National Natural Science Foundation of China under the Grant no. 12071368, and the Science and Technology Program of Shaanxi Province of China under the Grant no. 2019JM-573 and 2020JM-026. The second author was partially supported by the Austrian Science Fund FWF Project P 30405-N32.

(c) (1) $\Theta \Theta$ Licensed under the Creative Commons BY-NC-ND 4.0 International Public License. 
We study the balance and pattern distribution of the sequences $\left(s_{n}\right),\left(t_{n}\right)$ and $\left(u_{n}\right)$. For sets $\mathcal{S}$ with desirable pseudorandom properties, more precisely, sets with low correlation measures, we show the following:

(1) The sequence $\left(s_{n}\right)$ is (asymptotically) balanced and has uniform pattern distribution if $T$ is of smaller order of magnitude than $q$.

(2) The sequence $\left(t_{n}\right)$ is balanced and has uniform pattern distribution if $T$ is approximately $\left(1-\frac{1}{2^{1 /(m-1)}}\right) q$.

(3) The sequence $\left(u_{n}\right)$ is balanced and has uniform pattern distribution if $T$ is approximately $\frac{q}{2}$.

These results are motivated by earlier results for the sets of quadratic residues and primitive roots modulo a prime. We unify these results and derive many further (asymptotically) balanced sequences with uniform pattern distribution from pseudorandom subsets.

Communicated by Friedrich Pillichshammer.

\section{Introduction}

The balance and pattern distribution of several sequences derived from pseudorandom subsets of the finite field $\mathbb{Z}_{p}$ of prime order $p$ such as the set of quadratic residues and the set of primitive roots modulo $p$ have already been studied in the literature, see in particular [15, 16. In this paper we generalize the approach of [15, 16] for the sets of quadratic residues and primitive roots modulo $p$ to any pseudorandom subset. This unifies previous results and provides many new results for free.

\subsection{Fundamental examples}

More precisely, let $p>2$ be a prime, $q_{0}, q_{1}, \ldots, q_{\frac{p-3}{2}}$ be the quadratic residues modulo $p$ in increasing order and let $g_{0}, g_{1}, \ldots, g_{\varphi(p-1)-1}$ be the primitive roots modulo $p$ in increasing order, where $\varphi$ is Euler's totient function. 
Define the (finite) binary sequences $\left(s_{n}^{\prime}\right),\left(s_{n}^{\prime \prime}\right),\left(t_{n}^{\prime}\right),\left(t_{n}^{\prime \prime}\right),\left(u_{n}^{\prime}\right)$ and $\left(u_{n}^{\prime \prime}\right)$ by:

$$
\begin{aligned}
& s_{n}^{\prime} \equiv q_{n+1}-q_{n} \bmod 2, \quad n=0,1, \ldots, \frac{p-5}{2}, \\
& s_{n}^{\prime \prime} \equiv g_{n+1}-g_{n} \bmod 2, \quad n=0,1, \ldots, \varphi(p-1)-2 \text {, } \\
& t_{n}^{\prime}=\left\{\begin{array}{ll}
1 & \text { if } q_{n+1}-q_{n}=1, \\
0, & \text { otherwise, }
\end{array}=0,1, \ldots, \frac{p-5}{2},\right. \\
& t_{n}^{\prime \prime}=\left\{\begin{array}{ll}
1, & \text { if } g_{n+1}-g_{n}=1, \\
0, & \text { otherwise }
\end{array} \quad n=0,1, \ldots, \varphi(p-1)-2,\right.
\end{aligned}
$$

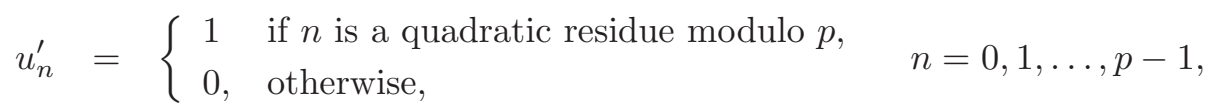

$$
\begin{aligned}
& u_{n}^{\prime \prime}=\left\{\begin{array}{ll}
1 & \text { if } n \text { is a primitive root modulo } p, \\
0, & \text { otherwise, }
\end{array} \quad n=0,1, \ldots, p-1 .\right.
\end{aligned}
$$

A (finite) binary sequence is balanced if the numbers of sequence elements equal to 0 and 1 differ by at most 1 . (Note that the term balanced is used with a different meaning in combinatorics on words, see for example [1, Definition 10.5.4].) A sequence of length $N$ is asymptotically balanced if this number is $o(N)$. Here

$$
f(n)=o(g(n)) \quad \text { if } \quad \lim _{n \rightarrow \infty} \frac{f(n)}{g(n)}=0 .
$$

A sequence $\left(s_{n}\right)$ of length $N$ has (asymptotically) uniform pattern distribution (of length $\ell$ ) if each pattern in $\{0,1\}^{\ell}$ of length $\ell$ appears $N 2^{-\ell}+o(N)$ times in the vector sequence

$$
\left(s_{n}, s_{n+1}, \ldots, s_{n+\ell-1}\right), \quad n=0,1, \ldots, N-\ell-1 .
$$

The extension of the terms balance and uniform pattern distribution to nonbinary sequences is obvious.

Since there are $(p-1) / 2$ quadratic residues and $\varphi(p-1)$ primitive roots modulo $p,\left(u_{n}^{\prime}\right)$ is balanced and $\left(u_{n}^{\prime \prime}\right)$ is asymptotically balanced if $\varphi(p-1)=\frac{p}{2}+o(p)$, which is true for example for Fermat primes, that is, $p$ is of the form $p=2^{k}+1$, and safe primes, that is, $(p-1) / 2$ is also a (Sophie Germain) prime. If $\varphi(p-1)$ is close to its maximum $(p-1) / 2$, then almost all quadratic non-residues are primitive roots and the sequences $\left(u_{n}^{\prime}\right)$ and $\left(u_{n}^{\prime \prime}\right)$ are essentially dual sequences with essentially the same uniform pattern distribution. Hence, there is no need to study $\left(u_{n}^{\prime \prime}\right)$ in this case. Ding [9] proved that $\left(u_{n}^{\prime}\right)$ has a uniform pattern distribution. 
Winterhof and Xiao (see [15]) showed that the sequence $\left(s_{n}^{\prime \prime}\right)$ is (asymptotically) balanced and has (asymptotically) uniform pattern distribution provided that $\varphi(p-1)=o(p)$ and proved in [16] that the sequence $\left(t_{n}^{\prime}\right)$ is essentially balanced and has uniform pattern distribution but the sequence $\left(s_{n}^{\prime}\right)$ is quite unbalanced. With the same methods one can show that $\left(t_{n}^{\prime \prime}\right)$ is balanced and has desirable pattern distribution if $\varphi(p-1)=\frac{p}{2}+o(p)$.

The desirable features of the above sequences are induced by certain pseudorandomness properties of the sets of quadratic residues and primitive roots modulo $p$. Our goal is to construct and analyze more such sequences derived from pseudorandom subsets of the residue class ring $\mathbb{Z}_{q}$ modulo $q$ which we identify with the integers between 0 and $q-1$, where $q$ may be composite. The desired pseudorandom properties are measured in terms of the correlation measures defined below.

\subsection{The general case}

Pseudorandom subsets have been studied in a series of papers (see [2], [4] 8, 12, 13]), in particular by Dartyge and Sárközy (partly with other coauthors). More precisely, let $q$ be a positive integer, $\mathcal{R} \subseteq \mathbb{Z}_{q}=\{0,1, \ldots, q-1\}$ and define

$$
f_{\mathcal{R}}(n)=\left\{\begin{aligned}
1-\frac{|\mathcal{R}|}{q}, & \text { for } n \in \mathcal{R}, \\
-\frac{|\mathcal{R}|}{q}, & \text { for } n \notin \mathcal{R} .
\end{aligned}\right.
$$

Dartyge and Sárközy [7] introduced the correlation measure $C_{k}(\mathcal{R}, q)$ of order $k$ of a subset $\mathcal{R}$ of $\mathbb{Z}_{q}$ as

$$
C_{k}(\mathcal{R}, q)=\max _{\substack{1 \leq M \leq q \\ 0 \leq d_{1}<d_{2}<\cdots<d_{k} \leq q-1}}\left|\sum_{n=0}^{M-1} f_{\mathcal{R}}\left(n+d_{1}\right) \cdots f_{\mathcal{R}}\left(n+d_{k}\right)\right| .
$$

Obviously, we have the trivial bound

$$
C_{k}(\mathcal{R}, q) \leq \min \{|\mathcal{R}|, q-|\mathcal{R}|\}
$$

The subset $\mathcal{R}$ is considered a pseudorandom subset of $\mathbb{Z}_{q}$ if $C_{k}(\mathcal{R}, q)$ is "small" (for all $k=1, \ldots, K$ for some sufficiently large $K$ ), that is, $o(\min \{|\mathcal{R}|, q-|\mathcal{R}|\}$ ). In particular, very small $\mathcal{R}$ are not considered pseudorandom and in some of our sequence constructions below we may restrict ourselves to sufficiently large $\mathcal{R}$. (Note that the expected size of a random $\mathcal{R}$ is $\frac{q}{2}$, its variance is $\frac{q}{4}$ and by Chebyshev's inequality the probability that a random subset $\mathcal{R}$ satisfies || $\mathcal{R}|-q / 2| \geq q^{1 / 2} \log q$ is $o(1)$ assuming that each element of $\mathbb{Z}_{q}$ belongs to $\mathcal{R}$ with probability $1 / 2$, that is, a binomial distribution.) However, we also provide a promising sequence construction from rather sparse subsets. For $\mathcal{R} \subseteq \mathbb{Z}_{q}$ we write 


\section{BALANCE AND PATTERN DISTRIBUTION FROM PSEUDORANDOM SUBSETS}

$$
C(\mathcal{R}, q, s)=\max _{1 \leq k \leq s} C_{k}(\mathcal{R}, q) .
$$

Dartyge and Sárközy also introduced the correlation measure of order $k$ for subsets of $\{1, \ldots, q\}$ in [5], that is, without reduction modulo $q$. Some bounds in the literature refer to this slightly different correlation measure. However, both measures are of the same order of magnitude and differ only by a multiplicative constant between 1 and 2 .

Throughout this paper we denote

$$
\mathcal{S}=\left\{x_{0}, x_{1}, \ldots, x_{T-1}\right\} \subseteq \mathbb{Z}_{q} \quad \text { with } \quad 0 \leq x_{0}<x_{1}<\cdots<x_{T-1} \leq q-1 .
$$

For given integers $M \geq 2$ and $m \geq 2$, let $\left(s_{n}\right)$ be the $M$-ary sequence defined by

$$
s_{n} \equiv x_{n+1}-x_{n} \bmod M, \quad n=0,1, \ldots, T-2,
$$

and let $\left(t_{n}\right)$ be the binary sequence defined by

$$
t_{n}= \begin{cases}1 & \text { if } 1 \leq x_{n+1}-x_{n} \leq m-1, \\ 0, & \text { otherwise }\end{cases}
$$

We also consider the characteristic sequence $\left(u_{n}\right)$ of $\mathcal{S}$,

$$
u_{n}=\left\{\begin{array}{ll}
1 & \text { if } n \in \mathcal{S}, \\
0, & \text { otherwise }
\end{array} \quad n=0,1, \ldots, q-1 .\right.
$$

Clearly, the sequence $\left(u_{n}\right)$ is balanced if and only if $T=\frac{q}{2}+o(q)$. We study the balance and pattern distribution of the sequences $\left(s_{n}\right)$ and $\left(t_{n}\right)$, and the pattern distribution of the sequence $\left(u_{n}\right)$ in Section 2 and give large families of examples in Section 3 .

We write

$$
f(n)=O(g(n)) \quad \text { or } \quad f(n) \ll g(n) \quad \text { if } \quad|f(n)| \leq c g(n)
$$

for some absolute constant $c>0$.

\section{Balance and pattern distribution}

For an $M$-ary sequence $\left(e_{n}\right)$ of length $T-1$ and $u \in\{1, \ldots, M\}$ let $N^{(u)}\left(e_{n}\right)$ denote the number of $n=0,1, \ldots, T-2$ with $e_{n} \equiv u \bmod M$. If

$$
N^{(u)}\left(e_{n}\right)=\left(\frac{1}{M}+o(1)\right) T \text { for all } u \in\{1, \ldots, M\},
$$

we say that $\left(e_{n}\right)$ is (asymptotically) balanced. 
In this section we study the balance of the sequences $\left(s_{n}\right)$ and $\left(t_{n}\right)$. We start with a preliminary result.

\subsection{A preliminary result}

Lemma 2.1. Let $\mathcal{R}$ be a subset of $\mathbb{Z}_{q}=\{0,1, \ldots, q-1\}$. Define

$$
c(i)= \begin{cases}+1, & i \in \mathcal{R}, \\ -1, & i \in \mathbb{Z}_{q} \backslash \mathcal{R} .\end{cases}
$$

For any positive integer $s$ with $1 \leq s \leq q$ and any $\left(\varepsilon_{0}, \ldots, \varepsilon_{s-1}\right) \in\{-1,+1\}^{s}$, we put

$$
\Gamma\left(\varepsilon_{0}, \ldots, \varepsilon_{s-1}\right)=\left|\left\{n=0,1, \ldots, q-s: c(n+i)=\varepsilon_{i}, i=0, \ldots, s-1\right\}\right| .
$$

Let $z=z\left(\varepsilon_{0}, \ldots, \varepsilon_{s-1}\right)$ be the number of $i$ with $\varepsilon_{i}=1, i=0, \ldots, s-1$. Then we have

$$
\Gamma\left(\varepsilon_{0}, \ldots, \varepsilon_{s-1}\right)=\left(\frac{|\mathcal{R}|}{q}\right)^{z}\left(1-\frac{|\mathcal{R}|}{q}\right)^{s-z} q+O\left(2^{s} C(\mathcal{R}, q, s)\right), \quad q \rightarrow \infty .
$$

Pr o of. Since otherwise the result is trivial we may assume $\mathcal{R} \neq \emptyset$ and $s \leq \log q$. Let $I \subseteq\{0, \ldots, s-1\}$ be the set of indices of size $z$ satisfying

Note that

$$
\varepsilon_{i}=\left\{\begin{aligned}
1, & i \in I \\
-1, & i \notin I
\end{aligned}\right.
$$

$$
f_{\mathcal{R}}(n)+\frac{|\mathcal{R}|}{q}= \begin{cases}1, & c(n)=1 \\ 0, & c(n)=-1\end{cases}
$$

where $f_{\mathcal{R}}(n)$ is defined by (11). Then

$$
\begin{aligned}
\Gamma\left(\varepsilon_{0}, \ldots, \varepsilon_{s-1}\right)= & \sum_{n=0}^{q-s} \prod_{i \in I}\left(f_{\mathcal{R}}(n+i)+\frac{|\mathcal{R}|}{q}\right) \prod_{i \notin I}\left(1-\frac{|\mathcal{R}|}{q}-f_{\mathcal{R}}(n+i)\right) \\
= & \left(\frac{|\mathcal{R}|}{q}\right)^{z}\left(1-\frac{|\mathcal{R}|}{q}\right)^{s-z}(q-s+1) \\
& +\sum_{\substack{H \subseteq I \\
J \subseteq\{0, \ldots, s-1\} \backslash I \\
H \cup J \neq \emptyset}}\left(\frac{|\mathcal{R}|}{q}\right)^{z-|H|}\left(1-\frac{|\mathcal{R}|}{q}\right)^{s-z-|J|} \\
& \times(-1)^{|J|} \sum_{n=0}^{q-s} \prod_{i \in H \cup J} f_{\mathcal{R}}(n+i) .
\end{aligned}
$$




\section{BALANCE AND PATTERN DISTRIBUTION FROM PSEUDORANDOM SUBSETS}

Since the absolute value of the sums over $n$ can be estimated by $C_{|H \cup J|}(\mathcal{R}, q) \leq$ $C(\mathcal{R}, q, s)$ and the number of pairs $(H, J)$ to be considered is $2^{s}-1$ we get the result.

\subsection{Balance of $\left(s_{n}\right)$}

Theorem 2.2. Let $M \geq 2$ and $T$ be integers with

$$
\frac{\log \log \log q}{\log \log q} \leq T \leq q .
$$

Let the subset $\mathcal{S}$ of size $T$ and the $M$-ary sequence $\left(s_{n}\right)$ be defined by (2) and (3), respectively. For any integer $u$ with $1 \leq u \leq M$ let $N^{(u)}\left(s_{n}\right)$ denote the number of $n=0,1, \ldots, T-2$ with $s_{n} \equiv u \bmod M$. Then we have

$$
\begin{aligned}
N^{(u)}\left(s_{n}\right)= & \left(\frac{\frac{T}{q}\left(1-\frac{T}{q}\right)^{u-1}}{1-\left(1-\frac{T}{q}\right)^{M}}+o(1)\right) T \\
& +O\left(M C(\mathcal{S}, q, M \log \log q)(\log q)^{M-2}\right), \quad q \rightarrow \infty .
\end{aligned}
$$

If $T=o(q)$, then we have

$$
\begin{aligned}
N^{(u)}\left(s_{n}\right)= & \left(\frac{1}{M}+o(1)\right) T \\
& +O\left(M C(\mathcal{S}, q, M \log \log q)(\log q)^{M-2}\right), \quad q \rightarrow \infty,
\end{aligned}
$$

and the sequence is (asymptotically) balanced if

$$
C(\mathcal{S}, q, M \log \log q)=o\left(\frac{T}{(\log q)^{M-2}}\right) .
$$

Proof. For integers $k \geq 0$ and $u$ with $1 \leq u \leq M$ put

$$
N_{k, u}=\Gamma(1, \underbrace{-1, \ldots,-1}_{M k}, \underbrace{-1, \ldots,-1}_{u-1}, 1) .
$$

Choose

$$
H=\lfloor\log \log q\rfloor-4
$$

and verify that for $T \geq \frac{\log \log \log q}{\log \log q} q$

$$
\begin{aligned}
\left(1-\frac{T}{q}\right)^{M(H+1)} & \leq\left(1-\frac{\log \log \log q}{\log \log q}\right)^{M \log \log q-4 M} \\
& \leq(\log \log q)^{-M}\left(1-\frac{\log \log \log q}{\log \log q}\right)^{-4 M}=o(1)
\end{aligned}
$$


where we used

$$
1-x \leq e^{-x}, \quad \text { and } \quad \frac{\frac{T}{q}\left(1-\frac{T}{q}\right)^{u-1}}{1-\left(1-\frac{T}{q}\right)^{M}} \leq 1, \quad 1 \leq T \leq q
$$

By Lemma 2.1 with $z=2$ and $s=M k+u+1 \leq M \log \log q, k=0,1, \ldots, H$, we have

$$
\begin{aligned}
N^{(u)}\left(s_{n}\right) \geq & \sum_{k=0}^{H} N_{k, u} \\
= & \sum_{k=0}^{H}\left(\left(\frac{T}{q}\right)^{2}\left(1-\frac{T}{q}\right)^{M k+u-1} q\right. \\
& \left.+O\left(2^{M k+u+1} C(\mathcal{S}, q, M k+u+1)\right)\right) \\
= & \left(\frac{\frac{T}{q}\left(1-\frac{T}{q}\right)^{u-1}}{\left.1-\left(1-\frac{T}{q}\right)^{M}+o(1)\right) T}\right) \\
& +O\left(2^{M H+M+1} C(\mathcal{S}, q, M H+M+1)\right) \\
= & \left(\frac{\frac{T}{q}\left(1-\frac{T}{q}\right)^{u-1}}{1-\left(1-\frac{T}{q}\right)^{M}+o(1)}\right) T \\
& +O\left(C(\mathcal{S}, q, M \log \log q)(\log q)^{M-2}\right) .
\end{aligned}
$$

Note that

$$
\sum_{u=1}^{M} \frac{\frac{T}{q}\left(1-\frac{T}{q}\right)^{u-1}}{1-\left(1-\frac{T}{q}\right)^{M}}=1
$$

Hence, for each $v=1, \ldots, M$ we have

$$
\begin{aligned}
N^{(v)}\left(s_{n}\right)= & T-1-\sum_{u \neq v} N^{(u)}\left(s_{n}\right) \\
\leq & T-(1+o(1)) T+O\left(M C(\mathcal{S}, q, M \log \log q)(\log q)^{M-2}\right) \\
& +\frac{\frac{T}{q}\left(1-\frac{T}{q}\right)^{v-1}}{1-\left(1-\frac{T}{q}\right)^{M}} T
\end{aligned}
$$

and the first result follows. 


\section{BALANCE AND PATTERN DISTRIBUTION FROM PSEUDORANDOM SUBSETS}

If $T=o(q)$, then we get

$$
\frac{\frac{T}{q}\left(1-\frac{T}{q}\right)^{u-1}}{1-\left(1-\frac{T}{q}\right)^{M}}=\frac{1+\sum_{j=1}^{u-1}\left(\begin{array}{c}
u-1 \\
j
\end{array}\right)\left(-\frac{T}{q}\right)^{j}}{M+\sum_{j=2}^{M}\left(\begin{array}{c}
M \\
j
\end{array}\right)\left(-\frac{T}{q}\right)^{j-1}}=\frac{1+o(1)}{M+o(1)}=\frac{1}{M}+o(1)
$$

and the second result follows.

\subsection{Balance of $\left(t_{n}\right)$}

Theorem 2.3. Let $T$ be an integer with $1 \leq T \leq q$. Let the subset $\mathcal{S}$ and the sequence $\left(t_{n}\right)$ be defined by (2) and (4), respectively. For $v \in\{0,1\}$ let $N^{(v)}\left(t_{n}\right)$ denote the number of $n=0,1, \ldots, T-2$ with $t_{n}=v$. Then we have

$$
N^{(v)}\left(t_{n}\right)=\left(1-\frac{T}{q}\right)^{(m-1)(1-v)}\left(1-\left(1-\frac{T}{q}\right)^{m-1}\right)^{v} T+O\left(2^{m} C(\mathcal{S}, q, m)\right),
$$

which shows that the sequence is (asymptotically) balanced if

$$
T=\left(1-\frac{1}{2^{1 /(m-1)}}\right) q+o(q) \quad \text { and } \quad C(\mathcal{S}, q, m)=o(T) .
$$

P r o of. By Lemma 2.1 we get

$$
\begin{aligned}
N^{(1)}\left(t_{n}\right) & =\sum_{u=1}^{m-1} \Gamma(1, \underbrace{-1, \ldots,-1}_{u-1}, 1) \\
& =\sum_{u=1}^{m-1}\left(\left(\frac{T}{q}\right)^{2}\left(1-\frac{T}{q}\right)^{u-1} q+O\left(2^{u+1} C(\mathcal{S}, q, u+1)\right)\right) \\
& =\left(1-\left(1-\frac{T}{q}\right)^{m-1}\right) T+O\left(2^{m} C(\mathcal{S}, q, m)\right)
\end{aligned}
$$

and

$$
N^{(0)}\left(t_{n}\right)=T-1-N^{(1)}\left(t_{n}\right)=\left(1-\frac{T}{q}\right)^{m-1} T+O\left(2^{m} C(\mathcal{S}, q, m)\right),
$$

which completes the proof of the first result.

The second result follows since

$$
\left(1-\frac{T}{q}\right)^{m-1}=\left(1-\left(1-\frac{T}{q}\right)^{m-1}\right)
$$

is equivalent to $T=\left(1-\frac{1}{2^{1 /(m-1)}}\right) q$. 


\subsection{Pattern distribution of $\left(s_{n}\right)$}

TheOREM 2.4. Let $M \geq 2$ and $T$ be integers with

$$
\frac{\log \log \log q}{\log \log q} q \leq T \leq q
$$

Let the subset $\mathcal{S}$ of size $T$ and the $M$-ary sequence $\left(s_{n}\right)$ be defined by (2) and (3), respectively. Let $\left(a_{0}, \ldots, a_{\ell-1}\right) \in\{1,2, \ldots, M\}^{\ell}$ be any pattern of fixed length $\ell \geq 1$. Let $N^{\left(a_{0}, \ldots, a_{\ell-1}\right)}\left(s_{n}\right)$ be the number of $n=0,1, \ldots, T-\ell-1$ with $s_{n+i}=a_{i}$ for $i=0, \ldots, \ell-1$. Then we have

$$
\begin{aligned}
N^{\left(a_{0}, \ldots, a_{\ell-1}\right)}\left(s_{n}\right)= & \left(\frac{\left(\frac{T}{q}\right)^{\ell}\left(1-\frac{T}{q}\right)^{a_{0}+\cdots+a_{\ell-1}-\ell}}{\left(1-\left(1-\frac{T}{q}\right)^{M}\right)^{\ell}}+o(1)\right)^{T} \\
& +O\left(M^{\ell} C(\mathcal{S}, q, \ell M \log \log q)(\log q)^{\ell M}\right),
\end{aligned}
$$

and the sequence of patterns of length $\ell$ is (asymptotically) balanced if

$$
T=o(q) \quad \text { and } \quad C(\mathcal{S}, q, \ell M \log \log q)=o\left(T /(\log q)^{\ell M}\right) .
$$

Proof. For non-negative integers $k_{0}, \ldots, k_{\ell-1}$ we put

$$
\begin{aligned}
& N_{k_{0}, \ldots, k_{\ell-1} ; a_{0}, \ldots, a_{\ell-1}}^{\prime} \\
& =\Gamma(\underbrace{1,-1, \ldots,-1}_{M k_{0}}, \underbrace{-1, \ldots,-1}_{a_{0}-1}, 1, \underbrace{-1, \ldots,-1}_{M k_{1}}, \underbrace{-1, \ldots,-1}_{a_{1}-1}, \ldots, 1, \\
& \underbrace{-1, \ldots,-1}_{M k_{\ell-1}}, \underbrace{-1, \ldots,-1}_{a_{\ell-1}-1}, 1) .
\end{aligned}
$$

Take

$$
H=\lfloor\log \log q\rfloor-2
$$

and recall from the proof of Theorem 2.2 that

$$
\left(1-\frac{T}{q}\right)^{M(H+1)}=o(1) \quad \text { for } \quad T \geq \frac{\log \log \log q}{\log \log q} q .
$$


By Lemma 2.1 with $s=M\left(k_{0}+k_{1}+\cdots+k_{\ell-1}\right)+a_{0}+a_{1}+\cdots+a_{\ell-1}+1 \leq$ $\ell M \log \log q$ and $z=\ell+1$ we get as in the proof of Theorem 2.2

$$
\begin{aligned}
& N^{\left(a_{0}, \ldots, a_{\ell-1}\right)}\left(s_{n}\right) \geq \sum_{k_{0}, k_{1}, \ldots, k_{\ell-1}=0}^{H} N_{k_{0}, \ldots, k_{\ell-1} ; a_{0}, \ldots, a_{\ell-1}}^{\prime} \\
& =\sum_{k_{0}, k_{1}, \ldots, k_{\ell-1}=0}^{H}\left(\left(\frac{T}{q}\right)^{\ell+1}\left(1-\frac{T}{q}\right)^{M\left(k_{0}+\cdots+k_{\ell-1}\right)+a_{0}+\cdots+a_{\ell-1}-\ell} q\right. \\
& +O\left(2^{M\left(k_{0}+\cdots+k_{\ell-1}\right)+a_{0}+\cdots+a_{\ell-1}+1}\right. \\
& \left.\left.C\left(\mathcal{S}, q, M\left(k_{0}+\cdots+k_{\ell-1}\right)+a_{0}+\cdots+a_{\ell-1}+1\right)\right)\right) \\
& =\prod_{i=0}^{\ell-1}\left(\frac{\frac{T}{q}\left(1-\frac{T}{q}\right)^{a_{i}-1}}{1-\left(1-\frac{T}{q}\right)^{M}}+o(1)\right) T \\
& +O\left(2^{M \ell(H+1)} C(\mathcal{S}, q, M \ell(H+1)+1)\right) \\
& =\left(\frac{\left(\frac{T}{q}\right)^{\ell}\left(1-\frac{T}{q}\right)^{a_{0}+\cdots+a_{\ell-1}-\ell}}{\left(1-\left(1-\frac{T}{q}\right)^{M}\right)^{\ell}}+o(1)\right) T \\
& +O\left((C(\mathcal{S}, q, \ell M \log \log q))(\log q)^{\ell M}\right) .
\end{aligned}
$$

From (6) we get

$$
\sum_{a_{0}, a_{1}, \ldots, a_{\ell-1}=1}^{M} \frac{\left(\frac{T}{q}\right)^{\ell}\left(1-\frac{T}{q}\right)^{a_{0}+\cdots+a_{\ell-1}-\ell}}{\left(1-\left(1-\frac{T}{q}\right)^{M}\right)^{\ell}}=\left(\sum_{u=1}^{M} \frac{\frac{T}{q}\left(1-\frac{T}{q}\right)^{u-1}}{1-\left(1-\frac{T}{q}\right)^{M}}\right)^{\ell}=1
$$

and the first result from

$$
N^{\left(a_{0}, \ldots, a_{\ell-1}\right)}\left(s_{n}\right)=T-\ell-\sum_{\left.\left(b_{0}, \ldots, b_{\ell-1}\right) \in\{1, \ldots, M\}^{\ell} \backslash\left\{\left(a_{0}, \ldots, a_{-1}\right)\right\}\right)} N^{\left(b_{0}, \ldots, b_{\ell-1}\right)}\left(s_{n}\right)
$$

and the lower bound on $N^{\left(a_{0}, \ldots, a_{\ell-1}\right)}\left(s_{n}\right)$. 
Finally, note that (7) implies

$$
\frac{\left(\frac{T}{q}\right)^{\ell}\left(1-\frac{T}{q}\right)^{a_{0}+\cdots+a_{\ell-1}-\ell}}{\left(1-\left(1-\frac{T}{q}\right)^{M}\right)^{\ell}}=\left(\frac{1}{M}+o(1)\right)^{\ell}=\frac{1}{M^{\ell}}+o(1)
$$

provided that $T=o(q)$.

\subsection{Pattern distribution of $\left(t_{n}\right)$}

TheOREM 2.5. Let $T$ be an integer with

$$
\frac{\log \log \log q}{\log \log q} q \leq T \leq q .
$$

Let the subset $\mathcal{S}$ of size $T$ and the sequence $\left(t_{n}\right)$ be defined by (2) and (41), respectively. Let $\left(b_{0}, \ldots, b_{\ell-1}\right) \in\{0,1\}^{\ell}$ be any pattern of length $\ell \geq 1$. Let $N^{\left(b_{0}, \ldots, b_{\ell-1}\right)}\left(t_{n}\right)$ be the number of $n=0,1, \ldots, T-\ell-1$ such that $t_{n+i}=b_{i}$ for $i=0, \ldots, \ell-1$. Then we have

$$
\begin{aligned}
& N^{\left(b_{0}, \ldots, b_{\ell-1}\right)}\left(t_{n}\right) \\
& =\left(\left(1-\frac{T}{q}\right)^{(m-1)\left(\ell-b_{0}-\cdots-b_{\ell-1}\right)}\left(1-\left(1-\frac{T}{q}\right)^{m-1}\right)^{b_{0}+\cdots+b_{\ell-1}}+o(1)\right) T \\
& \quad+O\left(2^{\ell(m-2)} C(\mathcal{S}, q, \ell \log \log q)(\log q)^{\ell}\right)
\end{aligned}
$$

and the sequence of patterns of length $\ell$ is (asymptotically) balanced if

$$
T=\left(1-\frac{1}{\left.2^{1 /(m-1}\right)}\right) q+o(q) \quad \text { and } \quad C(\mathcal{S}, q, \ell \log \log q)=o\left(T /(\log q)^{\ell}\right) .
$$

Proof. For positive integers $x_{0}, \ldots, x_{\ell-1}$ we put

$$
N_{x_{0}, \ldots, x_{\ell-1}}^{\prime \prime}=\Gamma(1, \underbrace{-1, \ldots,-1}_{x_{0}-1}, 1, \ldots, 1, \underbrace{-1, \ldots,-1}_{x_{\ell-1}-1}, 1) .
$$

Let $Z=b_{0}+\cdots+b_{\ell-1}$ be the number of $j=0, \ldots, \ell-1$ with $b_{j}=1$. Without loss of generality we may assume $b_{0}=\cdots=b_{Z-1}=1$ and $b_{Z}=\cdots=b_{\ell-1}=0$. We choose

$$
H=\lfloor\log \log q\rfloor-4
$$

and verify that for $T \geq \frac{\log \log \log q}{\log \log q} q$,

$$
\left(1-\frac{T}{q}\right)^{H}=o(1)
$$


By Lemma 2.1 with $s=x_{0}+\cdots+x_{\ell-1}+1 \leq \ell H+1 \leq \ell \log \log q$ and $z=\ell+1$ we have

$$
\begin{aligned}
& N^{\left(b_{0}, \ldots, b_{\ell-1}\right)}\left(t_{n}\right) \geq \sum_{x_{0}, \ldots, x_{z-1}=1}^{m-1} \sum_{x_{z}, \ldots, x_{\ell-1}=m}^{H} N_{x_{0}, \ldots, x_{\ell-1}}^{\prime \prime} \\
& =\sum_{x_{0}, \ldots, x_{z-1}=1}^{m-1} \sum_{x_{z}, \ldots, x_{\ell-1}=m}^{H}\left(\left(\frac{T}{q}\right)^{\ell}\left(1-\frac{T}{q}\right)^{x_{0}+\cdots+x_{\ell-1}-\ell} T\right. \\
& \left.+O\left(2^{x_{0}+\cdots+x_{\ell-1}} C(\mathcal{S}, q, \ell H+1)\right)\right) \\
& =\left(\left(1-\frac{T}{q}\right)^{m-1}-\left(1-\frac{T}{q}\right)^{H}\right)^{\ell-z}\left(1-\left(1-\frac{T}{q}\right)^{m-1}\right)^{z} T \\
& +O\left(2^{\ell(m+H+1)} C(\mathcal{S}, q, \ell \log \log q)\right) \\
& =\left(\left(1-\frac{T}{q}\right)^{(m-1)(\ell-z)}\left(1-\left(1-\frac{T}{q}\right)^{m-1}\right)^{z}+o(1)\right) T \\
& +O\left(2^{\ell(m-3)} C(\mathcal{S}, q, \ell \log \log q)(\log q)^{\ell}\right) \text {. }
\end{aligned}
$$

Note that

$$
\sum_{b_{0}, b_{1}, \ldots, b_{\ell-1}=0}^{1}\left(1-\frac{T}{q}\right)^{(m-1)\left(\ell-b_{0}-\cdots-b_{\ell-1}\right)}\left(1-\left(1-\frac{T}{q}\right)^{m-1}\right)^{b_{0}+\cdots+b_{\ell-1}}=1 .
$$

Thus we get Theorem 2.5.

\subsection{Pattern distribution of $\left(u_{n}\right)$}

The following result seems to be well-known at least for some special sets such as the set of primitive roots modulo a prime. However, for the convenience of the reader we add its short proof.

Theorem 2.6. Let $\mathcal{S} \subseteq \mathbb{Z}_{q}$ be of size $T$ and the sequence $\left(u_{n}\right)$ be defined by (5). Let $\left(b_{0}, \ldots, b_{\ell-1}\right) \in\{0,1\}^{\ell}$ be a pattern of length $\ell \geq 1$. Let $N^{\left(b_{0}, \ldots, b_{\ell-1}\right)}\left(u_{n}\right)$ be the number of $n=0,1, \ldots, q-\ell$ with $u_{n+i}=b_{i}$ for $i=0, \ldots, \ell-1$. Then we have

$$
N^{\left(b_{0}, \ldots, b_{\ell-1}\right)}\left(u_{n}\right)=\left(\frac{T}{q}\right)^{b_{0}+\cdots+b_{\ell-1}}\left(1-\frac{T}{q}\right)^{\ell-b_{0}-\cdots-b_{\ell-1}} q+O\left(2^{\ell} C(\mathcal{S}, q, \ell)\right)
$$

and the sequence of patterns of length $\ell$ is (asymptotically) balanced if

$$
T=\frac{q}{2}+o(q) \quad \text { and } \quad C(\mathcal{S}, q, \ell)=o(q) .
$$


Proof. Put $w=b_{0}+\cdots+b_{\ell-1}$ and $\varepsilon_{i}=(-1)^{b_{i}+1}$ for $i=0, \ldots, \ell-1$. By Lemma 2.1 we have

$$
\begin{aligned}
N^{\left(b_{0}, \ldots, b_{\ell-1}\right)}\left(u_{n}\right) & =\Gamma\left(\varepsilon_{0}, \ldots, \varepsilon_{\ell-1}\right) \\
& =\left(\frac{T}{q}\right)^{w}\left(1-\frac{T}{q}\right)^{\ell-w} q+O\left(2^{\ell} C(\mathcal{S}, q, \ell)\right)
\end{aligned}
$$

which implies the result.

\section{A primer on pseudorandom subsets}

Many pseudorandom subsets have been constructed and studied using number theoretic methods. We can derive large families of (asymptotically) balanced sequences with uniform pattern distribution from these pseudorandom subsets using our Theorems 2.2 to 2.6 .

Dartyge and Sárközy [6] constructed pseudorandom subsets using $d$ th power residues modulo $p$ for a divisor $d$ of $p-1$.

Proposition 3.1. Let $p \geq 2$ be a prime number, $d \mid p-1$ and let $f \in \mathbb{Z}_{p}[x]$ be a non-constant polynomial with no multiple roots in the algebraic closure $\overline{\mathbb{Z}}_{p}$ of $\mathbb{Z}_{p}$. Define

$$
\mathcal{R}=\left\{n: 0 \leq n \leq p-1, \exists y \in \mathbb{Z}_{p}^{*} \text { with } f(n) \equiv y^{d} \bmod p\right\} .
$$

Then we have

$$
|| \mathcal{R}\left|-\frac{p-m}{d}\right| \leq \frac{d-1}{d}(\operatorname{deg}(f)-1) p^{\frac{1}{2}},
$$

where $m \leq \operatorname{deg}(f)$ is the number of zeros of $f(x)$ in $\mathbb{Z}_{p}$.

Moreover, suppose that at least one of the following conditions is satisfied:

(i) $k \leq 2$;

(ii) $d$ is a prime divisor of $p-1$ and $(4 k)^{\operatorname{deg}(f)}<p$;

(iii) the polynomial $x^{p-1}+\cdots+x+1$ is irreducible in $\mathbb{Z}_{t}[x]$ for any prime divisor $t$ of $d$ and $\max (\operatorname{deg}(f), k)<p$.

Then we have

$$
C_{k}(\mathcal{R}, p) \ll(1+o(1))^{k}\left(1-\frac{1}{d}\right)^{k} \operatorname{deg}(f) k p^{\frac{1}{2}} \log p .
$$




\section{BALANCE AND PATTERN DISTRIBUTION FROM PSEUDORANDOM SUBSETS}

\section{REMARKS.}

(1) Note that (8) is slightly better than [6, (1.6)] and can be obtained from

$$
\left|V_{f}\right|-\frac{p-m}{d}=\frac{1}{d} \sum_{j=1}^{d-1} \sum_{n=0}^{p-1} \chi^{j}(f(n))
$$

and the Weil bound for complete multiplicative character sums, where $\chi$ is any character of order $d$ of $\mathbb{Z}_{p}^{*}$.

(2) In the special case $p>2, d=2$ and $f(x)=x$ the set $\mathcal{R}$ is the set of quadratic residues modulo $p$. Combining Theorem 2.2 with $M=2$, Theorem 2.5 with $m=2$ and Theorem 2.6. respectively, we recover essentially, that is, up to logarithmic and $o(1)$ terms, the following results:

(a) [16, Theorem 2] on the imbalance of the sequence $\left(s_{n}\right)$,

(b) [16, Theorem 3] on the uniform pattern distribution of $\left(t_{n}\right)$,

(c) [9, Proposition 2] on the uniform pattern distribution of the Legendre sequence $\left(u_{n}\right)$.

The reason for the slightly weaker results is our generic approach whereas in special cases we can optimize, for example, the choice of $H$ and can deal with complete sums over $\mathbb{Z}_{p}$ instead of incomplete ones.

(3) For sufficiently large $p, f(x)=x$ and, say,

$$
\log \log \log p \leq d \leq \frac{\log \log p}{\log \log \log p}
$$

combining Theorem 2.4 and Proposition 3.1 we see that the sequence $\left(s_{n}\right)$ has (asymptotically) a uniform pattern distribution.

Dartyge, Sárközy and Szalay [8] studied the pseudorandomness of subsets related to primitive roots modulo $p$.

Proposition 3.2. Let $p$ be an odd prime and let $\mathcal{G}_{p}$ be the set of the primitive roots modulo $p$. Let $s, r \in \mathbb{N}$ with $s|p-1, r| p-1$ and $f(x) \in \mathbb{Z}_{p}[x]$. Define the subset $\mathcal{R} \subseteq \mathbb{Z}_{p}$ by

$$
\mathcal{R}=\left\{g^{s}: g \in \mathcal{G}_{p}, \exists x \in \mathbb{Z}_{p}^{*} \text { with } f\left(g^{s}\right)=x^{r}\right\} .
$$

Then we have

$$
|\mathcal{R}|=\frac{1}{r} \varphi\left(\frac{p-1}{s}\right)+O\left(\operatorname{deg}(f) 2^{\omega\left(\frac{p-1}{s}\right)} p^{\frac{1}{2}} \log p\right),
$$

where $\omega(n)$ denotes the number of distinct prime factors of $n$. 
Suppose that $f(x)$ is irreducible over $\mathbb{Z}_{p}, \operatorname{deg}(f) \geq 2$ or $\operatorname{deg}(f)=r=1$. Then we have

$$
C_{k}(\mathcal{R}, p) \ll(1+o(1))^{k} k \operatorname{deg}(f) 2^{k \omega\left(\frac{p-1}{s}\right)} p^{\frac{1}{2}} \log p .
$$

\section{RemaRKS.}

(1) We have $2^{\omega(n)}=n^{o(1)}$, see for example [11], and thus

$$
2^{k \omega((p-1) / s)} p^{1 / 2} \log p \leq p^{\frac{1}{2}+o(1)} .
$$

(2) For $r=s=1$ and $f(x)=x$ we get the set of primitive roots modulo $p$. Combining Proposition 3.2 with Theorem 2.2, Theorem 2.4 and Theorem 2.6 with $M=m=2$, respectively, we recover essentially:

(a) [15, Theorems 1 and 2] on the (im-)balance and pattern distribution of the sequence $\left(s_{n}\right)$,

(b) [3, Theorem 1] on the pattern distribution of the sequence $\left(u_{n}\right)$.

Dartyge, Sárközy and Szalay [8] also studied the pseudorandomness of subsets defined by index properties, that is, elements with polynomial values in geometric progression.

Proposition 3.3. Let $p$ be a prime, $f(x) \in \mathbb{Z}_{p}[x]$ with $\operatorname{deg}(f) \geq 1, r \in \mathbb{Z}$, $s \in \mathbb{N}, s<p$. Define $\mathcal{R} \subseteq \mathbb{Z}_{p}$ by

$\mathcal{R}=\{n: 0 \leq n \leq p-1, \exists h \in\{r, r+1, \ldots, r+s-1\}$ with ind $f(n) \equiv h \bmod p\}$, where ind $m$ denotes the base $g$ index of $m$ to a fixed primitive root $g$ modulo $p$. Then we have

$$
|\mathcal{R}|=s+O\left(\operatorname{deg}(f) p^{\frac{1}{2}} \log p\right) .
$$

Moreover, suppose that at least one of the following conditions holds:

(i) $f$ is irreducible;

(ii) if $f$ has the factorization $f=f_{1}^{\alpha_{1}} \cdots f_{u}^{\alpha_{u}}$ where $\alpha_{i} \in \mathbb{N}$ and $f_{i}$ is irreducible over $\mathbb{Z}_{p}$, then there exists a $\beta$ such that exactly one or two $f_{i}$ 's have the degree $\beta$;

(iii) $k \leq 2$;

(iv) $(4 k)^{\operatorname{deg}(f)}<p$ or $(4 \operatorname{deg}(f))^{k}<p$.

Then we have

$$
C_{k}(\mathcal{R}, p) \ll(1+o(1))^{k} \operatorname{deg}(f) k 2^{k} p^{\frac{1}{2}}(\log p)^{k+1} .
$$




\section{BALANCE AND PATTERN DISTRIBUTION FROM PSEUDORANDOM SUBSETS}

\section{REMARKS.}

(1) This construction extends Gyarmati's earlier construction [10] for $r=1$ and $s=(p-1) / 2$.

(2) Propositions 3.1 and 3.2 provide only non-trivial constructions of size at most $(p-1) / 2$. Hence, Theorem 2.5 can guarantee uniform pattern distribution only for $m=2$. However, the size $s$ in the construction of Proposition 3.3 is very flexible and taking

$$
s=\left\lfloor\left(1-\frac{1}{2^{1 /(m-1)}}\right) p\right\rfloor
$$

we get a uniform pattern distribution for any $m \geq 2$.

Dartyge, Mosaki and Sárközy [4 presented the analogs for arithmetic progressions and inverses of arithmetic progressions.

Proposition 3.4. Assume that $p$ is an odd prime number, $f(x) \in \mathbb{Z}_{p}[x]$ is of degree $\operatorname{deg}(f) \geq 2$. Let $r \in \mathbb{Z}, s \in \mathbb{N}, s<p$. Define $\mathcal{R} \subseteq \mathbb{Z}_{p}$ by

$\mathcal{R}=\{n: 0 \leq n \leq p-1, \exists h \in\{r, r+1, \ldots, r+s-1\}$ with $f(n) \equiv h \bmod p\}$.

Then we have

$$
|\mathcal{R}|=s+O\left(\operatorname{deg}(f) p^{\frac{1}{2}} \log p\right)
$$

and for $k \leq \operatorname{deg}(f)-1$

$$
C_{k}(\mathcal{R}, p) \ll \operatorname{deg}(f) p^{\frac{1}{2}}(\log p)^{k+1} .
$$

Proposition 3.5. Assume that $p$ is an odd prime number, $r \in \mathbb{Z}, s \in \mathbb{N}, s<p$, $f(x) \in \mathbb{Z}_{p}[x]$ has no multiple root and $1 \leq \operatorname{deg}(f(x))<p$. Define $\mathcal{R} \subseteq \mathbb{Z}_{p}$ by

$$
\begin{array}{r}
\mathcal{R}=\{n: 0 \leq n \leq p-1, \operatorname{gcd}(f(n), p)=1, \exists h \in\{r, r+1, \ldots, r+s-1\} \\
\text { with } h f(n) \equiv 1 \bmod p\} .
\end{array}
$$

Then we have

$$
|\mathcal{R}|=s+O\left(\operatorname{deg}(f) p^{\frac{1}{2}} \log p\right)
$$

and for $k<\frac{p}{2 \operatorname{deg}(f)}$

$$
C_{k}(\mathcal{R}, p) \ll \operatorname{deg}(f) p^{\frac{1}{2}}(\log p)^{k+1} .
$$

Dartyge and Sárközy [7] presented constructions by using the argument of complex numbers, multiplicative and additive characters. 
Proposition 3.6. Let $p$ be a prime, $\chi$ a multiplicative character, $\psi$ a nontrivial additive character modulo $p, f(x), g(x) \in \mathbb{Z}_{p}[x]$ with $\operatorname{deg}(g) \geq 2$, and $\alpha, \beta$ real numbers with $\alpha<\beta \leq \alpha+1$. Define $\mathcal{R} \subseteq \mathbb{Z}_{p}$ by

$$
\begin{aligned}
\mathcal{R}=\{0 \leq n \leq p-1: \operatorname{gcd}(f(n), p)= & 1 \quad \text { and } \\
& 2 \pi \alpha \leq \arg (\chi(f(n)) \psi(g(n)))<2 \pi \beta\} .
\end{aligned}
$$

Then we have

$$
|\mathcal{R}|=(\beta-\alpha) p+O\left((\operatorname{deg}(f)+\operatorname{deg}(g)) p^{\frac{1}{2}} \log p\right)
$$

and for $k \leq \operatorname{deg}(g)-1$

$$
C_{k}(\mathcal{R}, p) \ll(1+o(1))^{k}(\operatorname{deg}(f)+\operatorname{deg}(g)) p^{\frac{1}{2}}(\log p)^{k+1} .
$$

\section{REMARKS.}

(1) This construction generalizes Dartyge's and Sárközy's construction [6] for $\chi$ being the Legendre symbol, $\psi$ trivial, $\alpha=-1 / 4$ and $\beta=1 / 4$, and extends Dartyge's, Mosaki's and Sárközy's construction [4] for $\chi$ trivial, $\psi$ canonical, $\alpha=\frac{r}{p}$ and $\beta=\frac{r+s-1}{p}$.

Let $p$ be a prime and let $n$ be an integer with $\operatorname{gcd}(n, p)=1$. The Fermat quotient $q_{p}(n)$ is defined as the unique integer with

$$
q_{p}(n) \equiv \frac{n^{p-1}-1}{p} \bmod p, \quad 0 \leq q_{p}(n) \leq p-1 .
$$

We also define

$$
q_{p}(n)=0 \quad \text { for } \operatorname{gcd}(n, p)>1 .
$$

The first author and Zhang [14] studied the pseudorandomness of subsets constructed by Fermat quotients using estimates for exponential sums and character sums with Fermat quotients.

Proposition 3.7. Let $p$ be an odd prime number, and $d \mid p-1$. Define $\mathcal{R} \subseteq \mathbb{Z}_{p^{2}}$ by

$$
\begin{aligned}
& \mathcal{R}=\left\{n: 0 \leq n \leq p^{2}-1, \exists y \quad\right. \text { such that } \\
& \left.\qquad 1 \leq y \leq p-1 \quad \text { and } \quad q_{p}(n) \equiv y^{d} \bmod p\right\} .
\end{aligned}
$$

Then we have

and

$$
|\mathcal{R}|=\frac{(p-1)^{2}}{d}
$$

$$
C_{k}\left(\mathcal{R}, p^{2}\right) \ll k p^{\frac{5}{3}} \text {. }
$$




\section{BALANCE AND PATTERN DISTRIBUTION FROM PSEUDORANDOM SUBSETS}

Proposition 3.8. Let $p$ be an odd prime number, and let $\mathcal{G}_{p}$ be the set of the primitive roots modulo $p$. Define $\mathcal{R} \subset \mathbb{Z}_{p^{2}}$ by

$$
\mathcal{R}=\left\{n: 0 \leq n \leq p^{2}-1, q_{p}(n) \in \mathcal{G}_{p}\right\} .
$$

Then we have

$$
|\mathcal{R}|=(p-1) \varphi(p-1)
$$

and

$$
C_{k}\left(\mathcal{R}, p^{2}\right) \ll k 2^{k \omega(p-1)} p^{\frac{5}{3}} .
$$

Now it is easy to plug the results of Propositions 3.1 to 3.8 in Theorems 2.2 to 2.6 to get many new results on balance and uniform pattern distribution of sequences of type $\left(s_{n}\right),\left(t_{n}\right)$ and $\left(u_{n}\right)$.

\section{REFERENCES}

[1] ALlOUCHE, J.-P.-SHALLIT, J.: Automatic sequences. Theory, applications, generalizations. Cambridge University Press, Cambridge, 2003.

[2] CHEN, Z.: Large families of pseudo-random subsets formed by generalized cyclotomic classes, Monatsh. Math. 161 (2010), no. 2, 161-172.

[3] COBELI, C.-ZAHARESCU, A.: On the distribution of primitive roots mod $p$, Acta Arith. 83 (1998), no. 2, 143-153.

[4] DARTYGE, C.-MOSAKI, E.-SÁRKÖZY, A.: On large families of subsets of the set of the integers not exceeding $N$, Ramanujan J. 18 (2009), no. 2, 209-229.

[5] DARTYGE, C.-SÁRKÖZY, A.: On pseudo-random subsets of the set of the integers not exceeding N, Period. Math. Hungar. 54 (2007), no. 2, 183-200.

[6] DARTYGE, C.-SÁRKÖZY, A.: Large families of pseudorandom subsets formed by power residues, Unif. Distrib. Theory 2 (2007), no. 2, 73-88.

[7] DARTYGE, C.-SÁRKÖZY, A.: On pseudo-random subsets of $\mathbb{Z}_{n}$, Monatsh. Math. 157 (2009), no. 1, 13-35.

[8] DARTYGE, C.-SÁRKÖZY, A.-SZALAY, M.: On the pseudo-randomness of subsets related to primitive roots, Combinatorica 30 (2010), no. 2, 139-162.

[9] DING, C.: Pattern distributions of Legendre sequences, IEEE Trans. Inform. Theory 44 (1998), no. 4, 1693-1698.

[10] GYARMATI, K.: On a family of pseudo-random binary sequences, Period. Math. Hungar. 49 (2004), no. 2, 45-63.

[11] HARDY, G. H.-WRIGHT, E. M.: An introduction to the theory of numbers. Fifth edition. The Clarendon Press, Oxford University Press, New York, 1979.

[12] LIU, H.-QI, Y.: On multi-dimensional pseudorandom subsets, J. Number Theory $\mathbf{1 8 1}$ (2017), 73-88.

[13] LIU, H.-SONG, E.: A note on pseudorandom subsets formed by generalized cyclotomic classes, Publ. Math. Debrecen 85 (2014), no. 3-4, 257-271. 


\section{HUANING LIU — ARNE WINTERHOF}

[14] LIU, H.-ZHANG, G.: Pseudo-random subsets constructed by using Fermat quotients, Publ. Math. Debrecen 94 (2019), no. 1-2, 55-74.

[15] WINTERHOF, A.-XIAO, Z.: Binary sequences derived from differences of consecutive primitive roots, IEEE Trans. Inform. Theory 67 (2021), no. 8, 5334-5338.

[16] WINTERHOF, A.-XIAO, Z.: Binary sequences derived from differences of consecutive quadratic residues, Adv. Math. Commun. to be published, doi: 10.3934/amc.2020100.

Received October 15, 2021

Accepted November 09, 2021

\section{Huaning Liu}

Research Center for

Number Theory and

its Applications

School of Mathematics

Northwest University

$X i$ 'an 710127

CHINA

E-mail: hnliu@nwu.edu.cn

\section{Arne Winterhof}

Johann Radon Institute

for Computational and

Applied Mathematics

Austrian Academy of Sciences

Altenberger Straße 69

4040 Linz

AUSTRIA

E-mail: arne.winterhof@oeaw.ac.at 